Pacific Journal of Mathematics

SOME REMARKS ON CONVOLUTION EQUATIONS FOR

( 


\title{
SOME REMARKS ON CONVOLUTION EQUATIONS FOR VECTOR-VALUED DISTRIBUTIONS
}

\author{
H. O. FATTORINI
}

Let $E, X$ be two complex Banach spaces, $(E ; X)$ the space of all linear bounded operators from $E$ into $X$ endowed with its usual norm. We denote by $\mathscr{D}^{\prime}(E)$ the space of distributions with values in $E$ defined in $-\infty<t<\infty$ and by $\mathscr{D}_{0}^{\prime}(E)$ the subspace of $\mathscr{D}^{\prime}(E)$ consisting of all $T \in \mathscr{D}^{\prime}(E)$ with support in $t \geqq 0$.

Given $P \in \mathscr{D}_{0}^{\prime}((X ; E))$ we examine the following problems.

(I). Does $P$ have a convolution inverse with support in $t \geqq 0$, that is, is there $S \in \mathscr{D}_{0}^{\prime}((E ; X))$ satisfying

$$
P * S=\delta \otimes I, S * P=\delta \otimes J
$$

where $I$ (resp. $J)$ denotes the identity operator in $E(\operatorname{resp} . X)$ and $\delta$ is the Dirac delta?

(II). In case the answer to (I) is affirmative, what properties of $S$ can be deduced from properties of $P$ and vice versa?

These problems have been exhaustively studied in the case where $P=\delta^{\prime} \otimes I-\delta \otimes A, A$ a closed, densely defined operator in $E$ and $X=D(A)$ with its graph norm. When $S$ is strongly continuous in $t \geqq 0$ then $S$ is a strongly continuous semigroup and $A$ its generator, characterized by the Hille-Yosida-Phillips theorem ([6], Ch. VIII); in the general case $\left(S \in \mathscr{D}_{0}^{\prime}((E ; X))\right) S$ is a distribution semigroup in the sense of Lions [15] and its generator $A$ is characterized by a result of Chazarain ([2], [3]; see also [19]). Several subcases and variants have been studied by Pazy [20], Barbu [1], Da Prato-Mosco [4], [5], Foias [12], Fujiwara [13], Yosida [25] and others (see [15] for the semigroup case). Similar problems for more general differential operators have also been considered; see for instance [8], [9], [10], [11], [24].

Our aim is to show here that many of the results just mentioned extend to the general case (with no restrictions on $P$, except perhaps on the support of $P$ or on its growth at infinity) although some interesting new phenomena appear. Motivation for this extension is provided by the fact that many state equations arising in applications are not purely differential; for instance equations describing the behavior of materials with memory (which appear in magnetic hysteresis, viscoelasticity, etc). We examine in detail in $\$ 8$ a heat equation proposed by Gurtin and Pipkin [14] where the temperature at a given time depends on the temperature history of the system. 
We shall assume throughout that the distribution $P$ in (1.1) belongs to the space $S_{0}^{\prime}((X ; E))$ of tempered, $(X ; E)$-valued distributions with support in $t \geqq 0$ (in fact, it is enough that $\exp (-\omega t) P \in \mathscr{S}_{0}^{\prime}((X ; E))$ ), since $\exp (-\omega t) P * \exp (-\omega t) S=\exp (-\omega t)(\delta \otimes I)=\delta \otimes I$ and a similar equation holds interchanging $P$ and $S)$. This can be dispensed with in some cases which are indicated later. Some of the results (for instance, Theorem 6.1) require that $P$ should have compact support; we write in this case $P \in \mathscr{E}_{0}^{\prime}((X ; E))$.

Existence of a solution to (1.1) is related to the possibility of solving the "Cauchy problem"

$$
P * U=T
$$

Here $T \in \mathscr{D}^{\prime}(E)$ is given with supp $(T)$ bounded below and $U \in \mathscr{D}^{\prime}(X)$ is required to satisfy

$$
\operatorname{supp}(U) \subseteq[\operatorname{supp}(T)]
$$

where [ $\cdot]$ indicates convex hull. This formulation is due to Lions [18]; note that if $P=\delta^{\prime} \otimes I-\delta \otimes A$ (1.2) reduces to

$$
\left(\frac{d}{d t}-A\right) U=T
$$

and if $U$ coincides, say, with a piecewise continuous function, then (1.3) simply states that $U$ has "zero initial value", i.e. $U=0$ for $t \leqq$ $\inf \operatorname{supp}(T)$. The relation mentioned above is established in $\$ 2$ where we also characterize those $P \in \mathscr{S}_{0}^{\prime}((X ; E))$ for which $(1.1)$ has a solution $S \in \mathscr{D}_{0}^{\prime}((E ; X))$ (we write then that $P \in \mathscr{D}_{0}^{\prime}((E ; X))^{-1}$ or simply $P \in$ $\left.\mathscr{D}_{0}^{\prime-1}\right)$. We look in $\S 3$ at conditions on $S=P^{-1}$ that imply exponential growth of $S$ at infinity and in $\S 4$ and $\S 5$ we establish a perturbation result and collect same observations on smoothness of solutions.

The case where $S$ is infinitely. differentiable is examined in $\$ 6$ and, finally, we set up in the last two sections a version of the Cauchy problem that applies to hereditary equations and study in this light some difference - differential equations and the Gurtin-Pipkin equation.

We note that, in most applications $X$ is a dense subspace of $E$ and the injection $i: X \rightarrow E$ is continuous (we denote this by $X \rightarrow E)$. However, this is unnecessary for most of the results and will only be assumed when explicity stated.

2. Existence of $S$. All the necessary information on vectorvalued distributions used in what follows can be found in Schwartz [22] and [23]. See also [21]. 
We start with two "local uniqueness" and "local existence" results. In them, we denote by $\mathscr{D}_{0}^{\prime}((-\infty, a) ;(E ; X))$ the space of all $(E ; X)$-valued distributions defined in $-\infty<t<a$ and vanishing for $t<0$.

Lemma 2.1. Let $S_{1}, S_{2} \in \mathscr{D}_{0}^{\prime}((-\infty, a) ;(E ; X))$ be two solutions of (1.1) in $t<a$. Then $S_{1}=S_{2}$ in $t<a$.

LEMMA 2.2. Assume that, for each $a>0$ we can find $S_{a} \in \mathscr{D}_{0}^{\prime}((-\infty, a) ;(E ; X))$ satisfying $(1.1)$ in $t<a$. Then there exists $S \in \mathscr{D}_{0}^{\prime}((E ; X))$ satisfying $(1.1)$ in $-\infty<t<\infty$.

The proof is immediate; in fact, $S_{2}=S_{2} * \delta=S_{2} *\left(P * S_{1}\right)=$ $\left(S_{2} * P\right) * S_{1}=\delta * S_{1}=S_{1}$ in $t<a$. To prove Lemma 2.2 define $S=S_{a}$ in $t<a$ and apply Lemma 2.1 to show that this definition makes sense.

Following Lions [18] and Chazarain [3] we say that the Cauchy problem for

$$
P * U=T
$$

is well set (in the sense of distributions) if

(i) For every $T \in \mathscr{D}^{\prime}(E)$ with support in $t \geqq a$ there exists a unique $U \in \mathscr{D}^{\prime}(X)$ with support in $t \geqq a$ satisfying (2.1).

(ii) The map $T \rightarrow U=M T$ from $\mathscr{D}^{\prime}(E)$ into $\mathscr{D}^{\prime}(X)$ defined by (2.1) is continuous if $\operatorname{supp}(T)$ is contained in a fixed interval $t \geqq a$.

The relation between (i), (ii) and the existence of a solution to (1.1) is given by

Lemma 2.3. Conditions (i) and (ii) hold for (2.1) if and only if (1.1) has a solution in $\mathscr{D}_{0}^{\prime}$.

The proof runs exactly like that of Corollaire 4.1 and Théorème 5.1 in [18], thus we only sketch it. If (1.1) has a solution then $U=S * T$ is a solution of (2.1) satisfying the requirements in (i). If $U_{1}, U_{2}$ are two such solutions, $P * U_{1}=P * U_{2}$ and the second equation (1.1) implies $U_{1}=$ $U_{2}$. Then (ii) follows from known results on continuity of the convolution product [22]. Conversely, if (i) and (ii) hold the map $U=M T$ defined by (2.1) must be given by $\mathcal{M T}=S * T, S \in \mathscr{D}_{0}^{\prime}((E ; X))$ ([18], Théorème 5.1) and is easy to see that $S$ must be a solution of (1.1).

We return now to (1.1). As in Schwartz [22] we shall indicate by $f(\hat{t})$ the function $t \rightarrow f(t)$ (or the distribution it defines) while $f(t)$ indicates the value of $f$ at $t$. Distributions will be sometimes written in "functional" notation: for instance, $\delta(\hat{t}-1)$ indicates the Dirac measure at $t=1$. We denote by $\mathfrak{B}(\lambda)$ or $\mathscr{L P}(\lambda)$ the Laplace transform of $P$ 
defined by $\mathfrak{P}(\lambda)=P(\exp (-\lambda \hat{t}))$. $\mathfrak{B}(\lambda)$ is defined in $\operatorname{Re} \lambda>0$, is analytic and has polynomial growth at infinity. It follows from analiticity of $\mathfrak{P}(\lambda)$ that $\mathfrak{P}(\lambda)^{-1}: E \rightarrow X$ exists and is bounded in an open (perhaps empty) subset $\rho(P)$ of the half-plane $\operatorname{Re} \lambda>0$.

Denote by $\mathscr{S}_{0, \omega}^{\prime}(E)$ the space of all distributions $T \in \mathscr{D}_{0}^{\prime}(E)$ with $\exp (-\omega \hat{t}) T \in \mathscr{S}_{0}^{\prime}(E)$.

Lemma 2.4. Let $P \in \mathscr{S}_{0}^{\prime}$. Then (1.1) has a solution $S \in \mathscr{Y}_{0, \omega}^{\prime}$ for some $\omega$ if and only if $\{\lambda ; \operatorname{Re} \lambda>\omega\} \subseteq \rho(P)$ and

$$
\left|\mathfrak{P}(\lambda)^{-1}\right|_{(E ; X)} \leqq C(1+|\lambda|)^{m} \quad(\operatorname{Re} \lambda>\omega)
$$

for some $C>0$ and some integer $m \geqq 0$.

The proof is immediate (see [18], Théorème 6.1). In fact, if $S \in \mathscr{S}_{0, \omega}^{\prime}$ we obtain from (1.1) that

$$
\mathscr{L P}(\lambda) \mathscr{L S}(\lambda)=I, \quad \mathscr{L S}(\lambda) \mathscr{L P}(\lambda)=J
$$

so that $\mathfrak{P}(\lambda)^{-1}=\mathscr{L S}(\lambda)$ and (2.2) follows from the fact that the Laplace transform of a distribution in $\mathscr{S}_{0, \omega}^{\prime}$ exists in $\operatorname{Re} \lambda>\omega$ and grows (at most) like a power of $|\lambda|$ at infinity (see [17]). On the other hand, any analytic function defined in $\operatorname{Re} \lambda>\omega$ and growing polynomially must be the Laplace transform of a distribution in $\mathscr{S}_{0, \omega}^{\prime}$ ([17]), so that, by virtue of (2.2) $\mathfrak{B}(\lambda)^{-1}=\mathscr{L} S(\lambda), S \in \mathscr{Y}_{0, \omega}^{\prime}$ and (2.3) is satisfied which, by uniqueness of Laplace transforms implies (1.1).

Theorem 2.5. Let $P \in \mathscr{Y}_{0}^{\prime}$. Then $P \in \mathscr{D}_{0}^{\prime-1}$ if and only if $\rho(P)$ contains a logarithmic region

$$
\Lambda(\alpha, \beta, \omega)=\{\lambda ; \operatorname{Re} \lambda \geqq \max (\alpha \log |\lambda|+\beta, \omega)\}
$$

where $\alpha, \beta, \geqq 0$ and

$$
\left|\mathfrak{B}(\lambda)^{-1}\right|_{(E ; X)} \leqq C(1+|\lambda|)^{m} \quad(\lambda \in \Lambda(\alpha, \beta, \omega))
$$

where $C>0$ and $m$ is an integer $\geqq 0$.

Proof. Let $\mathscr{H}_{a}$ be the set of all test functions in $\mathscr{D}$ which equal 1 in $t \leqq a, 0$ in $t \geqq 2 a$. If $\varphi \in \mathscr{H}_{a}$ we have

$$
P * \varphi S=\delta \otimes I-\Phi, \quad \varphi S * P=\delta \otimes J-\Psi
$$

where clearly $\Phi \in \mathscr{S}^{\prime}((E ; E)), \quad \Psi \in \mathscr{S}^{\prime}((X ; X))$ and (since 
$\Phi=P *(1-\varphi) S), \Phi=0$ for $t \leqq a$ and likewise $\Psi=0$ for $t \leqq a$. Accordingly,

$$
\Phi=f^{(p)}
$$

where $f$ is a $(E ; E)$-valued continuous function that vanishes for $t \leqq a$ and grows (at most) like a power of $t$ at infinity. This is easily seen to imply that

$$
|\mathscr{L} \Phi(\lambda)|_{(E, E)} \leqq C|\lambda|^{p-1} \exp (-a \operatorname{Re} \lambda) \quad(\operatorname{Re} \lambda>0,|\lambda| \geqq 1)
$$

If we now choose a positive $\gamma$ with $\gamma<1$ and take $\lambda$ in a logarithmic region (2.4) with $\beta=a^{-1} \log C \gamma^{-1}, \alpha=a^{-1}(p-1), \omega=1$ we have

$$
|\mathscr{L} \Phi(\lambda)|_{(E, E)} \leqq \gamma \quad(\lambda \in \Lambda(\alpha, \beta, \omega))
$$

On the other hand, since $\varphi S \in \mathscr{S}_{0}^{\prime}((E ; X))$,

$$
|\mathscr{L}(\varphi S)(\lambda)|_{(E, X)} \leqq C(1+|\lambda|)^{m} \quad(\operatorname{Re} \lambda>0)
$$

We take now the Laplace transform of the first equation (2.6) and avail ourselves of (2.8) which implies that $(I-\mathscr{L} \Phi(\lambda))^{-1}$ exists in $\Lambda(\alpha, \beta, \omega)$ and

$$
\left|(I-\mathscr{L} \Phi(\lambda))^{-1}\right|_{(E, E)} \leqq(1-\gamma)^{-1}
$$

there. We obtain

$$
\mathscr{L} P(\lambda) \mathscr{L}(\varphi S)(\lambda)(I-\mathscr{L} \Phi(\lambda))^{-1}=I \quad(\lambda \in \Lambda(\alpha, \beta, \omega))
$$

Operating now exactly in the same way with $\Psi$ we obtain (modifying if necessary the parameters $\alpha, \beta, \omega)$ that

$$
(I-\mathscr{L} \Psi(\lambda))^{-1} \mathscr{L}(\varphi S) \mathscr{L P}(\lambda)=I \quad(\lambda \in \Lambda(\alpha, \beta, \omega))
$$

which, together with (2.12) implies that $\mathfrak{B}(\lambda)^{-1}=$ $\mathscr{L}(\varphi S)(\lambda)(I-\mathscr{L} \Phi(\lambda))^{-1}$ exists in $\Lambda(\alpha, \beta, \omega)$, and in view of $(2.10)$ and (2.11), (2.5) holds. Conversely, assume the conditions in Theorem 2.5 are satisfied. Let $\Gamma$ be the boundary of the logarithmic region $\Lambda(\alpha, \beta+1, \omega+1)$ oriented from $-i \infty$ to $+i \infty$ and let $p>m$ a positive integer. Define

$$
\tilde{S}_{p}(t)=\frac{1}{2 \pi i} \int_{\Gamma} \lambda^{-p} e^{\lambda t} \mathfrak{P}(\lambda)^{-1} d \lambda
$$


Since the integrand in (2.14) is $O\left(|\lambda|^{\alpha t-p+m}\right)$ as $|\lambda| \rightarrow \infty, \tilde{S}_{p}$ is a continuous $(E ; X)$-valued function in $-\infty<t<(p-m-1) / \alpha$ and a standard deformation of contour argument shows that $\tilde{S}_{p}(t)=0$ for $t \leqq 0$. Now, since $P \in \mathscr{S}_{0}^{\prime}((X ; E))$ we can write

$$
P=f^{(q)}
$$

for some integer $q, f$ a continuous $(X ; E)$-valued function that vanishes for $t \leqq 0$ and is $O\left(|t|^{k}\right)$ as $t \rightarrow \infty$. We have

$$
\left(f * \tilde{S}_{p}\right)(t)=\frac{1}{2 \pi i} \int_{\Gamma} \lambda^{-p}\left\{e^{\lambda t} \int_{0}^{t} e^{-\lambda \eta} f(\eta) d \eta\right\} \mathfrak{P}(\lambda)^{-1} d \lambda
$$

$$
(-\infty<t<(p-m-1) / \alpha)
$$

where we have made the change of variables $t-s=\eta$ in the inner integral (the change of order of integration is easily justified from observing that the integrand is $O\left(|\lambda|^{\alpha s+p+m}\right)$ as $\left.|\lambda| \rightarrow \infty\right)$. Now, if

$$
\nu(\lambda)=e^{\lambda t} \int_{t}^{\infty} e^{-\lambda \eta} f(\eta) d \eta
$$

it is plain that $|\nu(\lambda)|=O\left(|\lambda|^{-1}\right)$ as $|\lambda| \rightarrow \infty, \operatorname{Re} \lambda>0$. Then it follows from another deformation of contour argument that the upper limit of integration in the inner integral in (2.15) can be changed to $+\infty$. Doing this and using the fact that $\mathfrak{P}(\lambda)=\lambda^{a} \mathscr{L} f(\lambda)$ we see that

$$
\left(f * \tilde{S}_{p}\right)(t)=t^{p+q-1} h(t) I \quad(t<(p-m-1) / \alpha)
$$

where $h$ is the Heaviside function. Differentiating $p+q$ times (2.16) we have

$$
P * S_{p}=\delta \otimes I \quad(t<(p-m-1) / \alpha)
$$

where we have set $S_{p}=\tilde{S}_{p}^{(p)}$. Operating in the same way we obtain

$$
S_{p} * P=\delta \otimes J \quad(t<(p-m-1) / \alpha
$$

and then, in view of the arbitrariness of $P$ the result follows from Lemma 2.2 .

Lemma 2.4 was proved by Lions [18] for the case $P=$ $\delta^{\prime} \otimes I-\delta \otimes A, X=D(A), X$ equipped with the graph norm; note that in these conditions we have $\mathfrak{B}(\lambda)^{-1}=R(\lambda ; A)$ and $\rho(P)=\rho(A)$. Theorem 2.5 was proved by Foias [12] in the same case under the 
assumption that $E$ is a Hilbert space and $A$ is normal and in all generality by Chazarain [2], [3] where $P$ is allowed to be a linear combination of derivatives (possibly of fractional order) of $\delta$. Note that in these results estimates of the type of $(2.5)$ are given in the $(E ; E)$-norm rather than the $(E ; X)$-norm as here; however, since

$$
\begin{aligned}
|R(\lambda ; A)|_{(E ; D(A))} & \leqq|R(\lambda ; A)|_{(E ; E)}+|A R(\lambda ; A)|_{(E ; E)} \\
& \leqq 1+\left.(1+|\lambda|) R(\lambda ; A)\right|_{(E ; E)}
\end{aligned}
$$

and $|R(\lambda ; A)|_{(E ; E)} \leqq|R(\lambda ; A)|_{(E, D(A))}$, the two norms can be interchanged. The same comment applies to all results involving $P=$ $\delta^{\prime} \otimes I-\delta \otimes A$ like Theorem 6.1.

REMARK 2.6. Theorem 2.5 can be extended - if in a somewhat awkward form - to the case $P \in \mathscr{D}_{0}^{\prime}$. In fact, let $\mathscr{H}=\cup_{a>0} \mathscr{H}_{a}$. Then we have

Theorem 2.7. Let $P \in \mathscr{D}_{0}^{\prime}$. Then $P \in \mathscr{D}_{0}^{\prime-1}$ if and only if $\varphi P \in$ $\mathscr{D}_{0}^{\prime-1}$ for all $\varphi \in \mathscr{H}$.

Proof. Let $\varphi \in \mathscr{H}_{a}$. If $\varphi P \in \mathscr{D}_{0}^{\prime-\mathrm{i}}$ then there exists $S_{\varphi} \in \mathscr{D}_{0}^{\prime}$ with $\varphi P * S_{\varphi}=\delta \otimes I, S_{\varphi} * \varphi P=\delta \otimes J$. But $\varphi P=P$ in $t \leqq a$, so $P * S_{\varphi}=$ $\delta \otimes I, S_{\varphi} * P=\delta \otimes J$ in $t<a$ and Lemma 2.2 applies. Conversely, if $P \in \mathscr{D}_{0}^{\prime-1}$ let $S=P^{-1}$. Then, if $\varphi \in \mathscr{H}_{a}$ we have $\varphi P * \varphi S=\delta \otimes I-\Phi$, $\varphi S * \Phi P=\delta \otimes I-\Psi$ where

$$
\Phi=\varphi P * \varphi S-\delta \otimes I=(1-\varphi) P *(1-\varphi) S-(1-\varphi) P * S-P *(1-\varphi) S
$$

belongs to $\mathscr{S}_{0}^{\prime}((E ; E))$ and vanishes in $t \leqq a$ and the same holds for $\Psi$. We can then proceed just as in the proof of the first half of Theorem 2.5 to show that $\varphi P$ satisfies the assumptions in the theorem and hence belongs to $\mathscr{D}_{0}^{\prime-1}$.

3. Exponential growth at infinity. We consider here the following problem.

III. Let $P \in \mathscr{D}_{0}^{\prime-1}$. What conditions on $P$ (or $S$ ) will imply that

$$
S \in \mathscr{Y}_{0, \omega}^{\prime}
$$

for some $\omega$ ?

We examine first the case $\operatorname{dim} E<\infty$ (note that if there is in this case a $P \in \mathscr{D}_{0}^{\prime-1}((E ; X)), \mathfrak{P}(\lambda): X \rightarrow E$ is invertible for some $\lambda$ and then $\operatorname{dim} X=\operatorname{dim} E$; we may then assume that $X=E$ ). Even in the case 
$\operatorname{dim} E=1$ there are $P \in \mathscr{D}_{0}^{\prime-1}$ where $P^{-1}$ does not satisfy (3.1); for instance, if $P=\delta(\hat{t})-\delta^{\prime}(\hat{t}-1)$,

$$
S=P^{-1}=\delta(\hat{t})+\delta^{\prime}(\hat{t}-1)+\delta^{\prime \prime}(\hat{t}-2)+\cdots
$$

which does not satisfy (3.1) for any $\omega$. On the other hand, if $P=$ $\delta^{\prime} \otimes I-\delta \otimes A$, then $P \in \mathscr{D}_{0}^{\prime-1}, S(t)=h(t) \exp (t A)$ and

$$
|S(t)| \leqq C e^{\omega t} \quad(t \geqq 0)
$$

for some $C, \omega \geqq 0$. This holds also for a more general class of distributions $P$; in fact, we have

LEMMA 3.1. Let

$$
P=\delta^{\prime} \otimes I-\mu
$$

$\mu$ a measure with values in $(E ; E)$ such that $|\mu|[0, \infty]=\omega<\infty$. Then $P \in \mathscr{D}_{0}^{\prime-1}$ and $S$ satisfies (3.2).

Proof. Define, for $\alpha>0$

$$
Y_{\alpha}(t)=t^{\alpha-1} / \Gamma(\alpha) \quad(t>0), \quad Y_{\alpha}(t)=0 \quad(t<0) .
$$

Then $Y_{\alpha}$ defines a distribution in $\mathscr{S}_{0}^{\prime}$ that can be continued anallytically to all complex $\alpha$ (see Schwartz [21]) and satisfies $Y_{\alpha} * Y_{\beta}=Y_{\alpha+\beta}, Y_{\alpha}^{\prime}=Y_{\alpha-1}$; moreover, $Y_{-m}=\delta^{(m)}$ for $m \geqq 0$.

We consider now the series

$$
S(t)=\sum_{n=0}^{\infty} Y_{n+1} * \mu^{* n}
$$

where the exponent $* n$ indicates convolution power. Note first that, since $Y_{n+1}, n>0$ is continuous in $-\infty<t<\infty$ each term in (3.4) is continuous. Since $\left|\mu^{* n}\right|[0, \infty]<\omega^{n}, \quad\left(Y_{n+1} * \mu^{* n}\right)(t)<t^{n} \omega^{n} / n !$ This shows that the series (3.4) is uniformly convergent on compacts and direct term-by-term computation shows that $S=P^{-1}$. Clearly $S$ satisfies (3.2) with $C=1$.

We note that, as a byproduct of Lemma 3.1 we obtain that $P^{-1}$ is continuous whenever $P$ is of the form (3.3) (the condition $|\mu|[0, \infty]<\infty$ is actually unnecessary, as we shall see later). Note that the result can be easily extended to $P=\delta^{(n)} \otimes I-Y_{-(n-1)} * \mu$. When $\operatorname{dim} E=\infty$ the situation is considerably more complex. In fact, even in the case $P=$ $\delta^{\prime} \otimes I-\delta \otimes A, S$ may not satisfy even (3.1) (see a general class of 
examples in Foias [12]). However, it is true in general that "if $P \in \mathscr{D}_{0}^{\prime-1}$ and $S$ is smooth enough, then (3.1) holds". To explain what we mean by this, consider again the case $P=\delta \otimes I-\delta \otimes A \in \mathscr{D}_{0}^{\prime-1}$ but assume that $S$ is strongly continuous in $t \geqq 0$, that is, assume that $A$ is the infinitesimal generator of the semigroup $S$. Then it is known that (3.2) holds. This result can be generalized to $P=\delta^{(n)} \otimes I-\delta \otimes A$ ([8], [10]). Assuming only that $P=\delta^{\prime} \otimes I-\delta \otimes A \in \mathscr{D}_{0}^{\prime-1}$ but requiring $S$ to be uniformly $C^{\infty}$ in $t>0$ Barbu [1] has shown that (3.1) holds. All these results are particular cases of the following.

TheOREM 3.2. Let $P \in \mathscr{D}_{0}^{\prime-1}$. Assume that, for some $\varphi \in \mathscr{H}$, $P * \varphi S-\delta \otimes I=P *(1-\varphi) S=Q$ is strongly continuous in $t>0$ and

$$
\int_{0}^{\infty} e^{-\omega t}|Q(t)| d t<\infty
$$

for some $\omega>0$. Then (3.1) holds.

(Note that the integral in (3.5) makes sense, as $|Q(t)|=$ $\sup \{|\mathrm{Q}(\mathrm{t}) \mathrm{u}| ;|\mathrm{u}| \leqq 1\}$ is lower semicontinuous, hence measurable).

Proof. Since $Q$ has support in $t \geqq a, Q^{* n}$ has support in $t>n a$ and the series

$$
M=Q-Q^{* 2}+Q^{* 3}-\cdots
$$

converges in $\mathscr{D}^{\prime}((E: E)$ ) (we don't use here any of the hypotheses in Theorem 3.2). Now, it is easy to see by direct term-by-term computation that, if $\tilde{S}=\varphi S *(\delta \otimes I-\mathcal{M})$ then $P * \tilde{S}=\delta \otimes I, \tilde{S} * P=\delta \otimes J$ so that

$$
\tilde{S}=\varphi S *(\delta \otimes I-\mathcal{M})
$$

It is not difficult to see (as in [6], Theorem VIII. 1.19) that $Q * Q$, $Q * Q * Q, \cdots$ etc. are all strongly continuous if $Q$ is. Taking $\omega$ large enough in (3.5) we may assume the integral equals $\gamma<1$ and, since

$$
e^{-\omega \hat{t}} Q^{* n}=\left(e^{-\omega \hat{t}} Q\right)^{* n}
$$

we obtain from Young's inequality, inductively, that

$$
\int_{0}^{\infty} e^{-\omega \hat{t}}\left|Q^{* n}(t)\right| d t<\gamma^{n} \quad(n \geqq 1)
$$


which implies that $\mathcal{M}$, which is also a $(E ; E)$-valued function (recall that the sum (3.6) is locally finite) satisfies

$$
\int_{0}^{\infty} e^{-\omega t}|\mathcal{M}(t)| d t \leqq(1-\gamma)^{-1}
$$

and thus belongs to $\mathscr{Y}_{0, \omega}^{\prime}$. Then, as $\varphi S \in \mathscr{S}_{0}^{\prime}$ it follows from (3.7) that $S$ belongs to $\mathscr{S}$ as well, which ends the proof of Theorem 3.5.

Remark 3.3. Let $P \in \mathscr{D}_{0}^{\prime-1}$ have compact support and assume that $S$ is a $C^{\infty}(E ; X)$-valued function for $t>b$. Then it is clear that, if $\varphi \in \mathscr{H}_{a}$ with $a>b, Q=P *(1-\varphi) S$ is a $C^{\infty}(E ; X)$ valued function for all $t$ and has compact support so that (3.5) is satisfied. It follows then from Theorem 3.2 that $S \in \mathscr{S}_{\omega}^{\prime}$ for some $\omega$. If $P \in \mathscr{Y}_{0}^{\prime}$, which falls short of (3.5), we don't know whether (3.1) must necessarily hold.

The requirement that $S$ be $C^{\infty}$ can be relaxed; in fact, if $P=\mu^{(m)}$ where $\mu$ is a $(X ; E)$-valued measure it is enough to require that $S$ should be $C^{(m)}$ for $t$ large enough. In some cases this requirement can be further relaxed; for instance, if $P=\delta^{\prime} \otimes I-\delta \otimes A$ with $A$ densely defined and closed, $X=D(A)$ with the graph norm and $S$ is merely strongly continuous (as an $(E ; E)$-valued function) for $t>b$ then Theorem 3.2 applies; in fact, $P * \varphi S-\delta \otimes I=\varphi^{\prime} S$.

REMARK 3.4. We note that the result of Theorem 3.2 holds if we replace $Q$ by $R=\varphi S * P-\delta \otimes J=(1-\varphi) S * P$. In this case we define

$$
\mathcal{N}=R-R^{* 2}+R^{* 3}-\cdots
$$

and (3.7) is replaced by

$$
S=(\delta \otimes I-\mathcal{N}) * \varphi S
$$

4. Smooth solutions. If $P=\delta^{\prime} \otimes I-\delta \otimes A \in \mathscr{D}_{0}^{\prime-1}$ it is known (see [18]) that if $u=S(\varphi) v, \varphi$ a test function with support in $t>0$ or, more generally, if $u \in D\left(A^{\infty}\right)=\bigcap_{n=1}^{\infty} D\left(A^{n}\right)$, then $S u$ coincides in $t>0$ with a function with values in $X$, infinitely differentiable in $t \geqq 0$ (See also [10]). We may state loosely this result as "smooth initial data produce smooth solutions". The situation becomes considerably more complicated in the general case, as the one-dimensional example $P=$ $\delta^{\prime}(\hat{t})-\delta^{\prime}(\hat{t}-1)$ shows; for in this case

$$
S(t)=\left\{\begin{array}{l}
1(2 k<t \leqq 2 k+1) \\
0(2 k+1<t \leqq 2 k+2)
\end{array}\right.
$$


(by modification of this example one may construct a $P \in \mathscr{D}_{0}^{\prime-1}$, this time in infinite dimensional space, where $S u$ is everywhere discontinuous for all $u \in E)$. Even in the less artificial difference-differential case $P=\delta^{\prime}(\hat{t})-\delta(\hat{t}-1)$ we have

$$
S(t)=Y_{1}(t)+Y_{2}(t-1)+Y_{3}(t-2)+\cdots
$$

which is merely continuous (although, it becomes smoother as $t \rightarrow \infty)$. This kind of situation arises in more generality; in fact,

Lemma 4.1. Let $\operatorname{dim} E<\infty$,

$$
P=\delta^{\prime} \otimes I-\mu \in \mathscr{D}_{0}^{\prime-1}
$$

$\mu$ a measure with values in $(E ; E)$. Then, if $S=P^{-1}, S$ is continuous in $t \geqq 0$. Hence

Proof. As in Lemma 3.1, $X=E$. We have $S^{\prime}=\mu * S+\delta \otimes I$.

$$
S=\mu * Y_{1} * S+Y_{1} .
$$

Replacing $S$ in the right-hand side of (4.2) by its left-hand side and iterating, we obtain

$$
S=\mu^{* n} * Y_{n} * S+\sum_{k=1}^{n} \mu^{*(k-1)} * Y_{n}
$$

But $S$ is, locally, the derivative of a continuous function, thus $Y_{n} * S$ will be continuous on any given compact if $n$ is large enough. This proves Lemma 4.1 (note that the proof can be generalized to the case $\left.P=\delta^{(n)} \otimes I-Y_{-(n-1)} * \mu\right)$.

When $\operatorname{dim} E=\infty$ the situation becomes more complicated. Assume (in the case $X \rightarrow E$ ) we call an element $u \in X$ "smooth" if $P u \in$ $\mathscr{D}_{0}^{\prime}(X ; X), P * P u \in \mathscr{D}_{0}^{\prime}(X, X), P * P * P u \in \mathscr{D}_{0}^{\prime}(X ; X), \cdots$ which would be a generalization of the condition $u \in D\left(A^{\infty}\right)$ in the case $P=$ $\delta^{\prime} \otimes I-\delta \otimes A$. Then it is possible to show that $S u$ is continuous for $t \geqq 0$ by using the same argument as in Lemma 4.1 if $P$ is of the form (4.1). However, even in the purely differential case, there may be no other smooth elements that $u=0$, as the following example shows. Let $P=\delta^{\prime \prime} \otimes I-\delta^{\prime} \otimes A-\delta \otimes B$ with $A, B$ closed, $X=D(A) \cap D(B)$ with $|u|_{X}=|u|+|A u|+|B u|$. Then it is not difficult to see that $u$ is smooth if and only if $u \in D\left(A_{1} A_{2} \cdots A_{n}\right)$ for all $n \geqq 1$, where $A_{i}=A$ or 
$A_{i}=B$. Let now $E=H$ be a Hilbert space, $A$ an unbounded infinitesimal generator, $B u=\left(u, u_{0}\right) u_{0}$ where $u_{0} \notin D(A)$. Since

$$
\mathfrak{P}(\lambda)^{-1}=\frac{1}{\lambda} \sum_{n=0}^{\infty}\left[\frac{1}{\lambda} R(\lambda ; A) B\right]^{n} R(\lambda ; A)
$$

exists for $\operatorname{Re} \lambda$ large enough and is bounded there, $P \in \mathscr{\mathscr { P }}_{0}^{\prime-1}$ by Lemma 2.4. But $D(A B)=\{0\}$, so there cannot be nonzero smooth elements. It would be interesting to know conditions on $P$ (for instance, $P(\varphi) P(\psi)=P(\psi) P(\varphi))$ that imply denseness of smooth elements.

5. A perturbation result. The following theorem is based on a classical result on perturbation of strongly continuous semigroups ([6], Theorem VIII. 1.19) and thus the proof will only be sketched.

THEOREM 5.1. Let $P \in \mathscr{D}_{0}^{\prime} \cap \mathscr{D}_{0}^{\prime-1}$ and let $P_{1} \in \mathscr{D}^{\prime}((X ; E))$ be such that $(a)$ if $S=P^{-1}, P_{1} * S$ is an $(E ; E)$-valued function strongly continuous for $t>0$ and $(b)$

$$
\int_{0}^{\infty} e^{-\omega t}\left|\left(P_{1} * S\right)(t)\right| d t<\infty
$$

for some $\omega>0$. Then $P+P_{1} \in \mathscr{D}_{0}^{\prime-1}$.

The proof is carried out much in the same way as that of Theorem 3.2. As in [6], Lemma VIII. 1.21 we prove first that $\left(P_{1} * S\right)^{* 2}$, $\left(P_{1} * S\right)^{* 3}, \cdots$ are strongly continuous for $t>0$. There we choose $\omega$ so large that the integral (5.1) equals $\gamma<1$ and prove by means of Young's inequality that

$$
\int_{0}^{\infty} e^{-\omega t}\left|\left(P_{1} * S\right)^{* n}(t)\right| d t \leqq \gamma^{n} \quad(n \geqq 1)
$$

Now, it is easy to show that (5.2) implies that the partial sums of the series

$$
\mathcal{U}=P_{1} * S-\left(P_{1} * S\right)^{* 2}+\cdots
$$

are a Cauchy sequence in $\mathscr{S}_{0, \omega}^{\prime}((E ; E))$ so that the series is convergent. Again, direct term-by-term computation shows that

$$
\begin{aligned}
& \left(P+P_{1}\right) *(S *(\delta \otimes I-\mathcal{U}))=\delta \otimes I \\
& (S *(\delta \otimes I-\mathcal{U})) *\left(P+P_{1}\right)=\delta \otimes J
\end{aligned}
$$

which ends the proof the Theorem 5.1. 
REMARK 5.2. A similar result holds if we replace $P_{1} * S$ by $S * P_{1}$; the requirements are that $S * P_{1}$ should coincide for $t>0$ with an $(X ; X)$-valued continuous function and (5.1) - where the norm is now the $(X ; X)$ - norm should be satisfied. In this case we obtain $\left(P+P_{1}\right)^{-1}=(\delta \otimes I-\mathscr{V}) * S$, where

$$
\mathscr{V}=S * P_{1}-\left(S * P_{1}\right)^{* 2}+\cdots
$$

Remark 5.3. Generally speaking, Theorem 5.1 yields better results when $S$ is not too singular, for otherwise the conditions on $P_{1}$ become excessively stringent. A particularly important case is that where $S$ itself is strongly continuous and

$$
|S(t)| \leqq C e^{\omega t} \quad(t \geqq 0)
$$

for some $C, \omega>0$; evidently, we may assume that this $\omega$ is the same as the one in (5.2) and obtain that

$$
\begin{aligned}
& \left|S *\left(P_{1} * S\right)^{* n}(t)\right| \\
\leqq & e^{\omega t} \int_{0}^{t} e^{-\omega(t-s)}|S(t-s)| e^{-\omega s}\left|\left(P_{1} * S\right)^{* n}(t)\right| d t \\
\leqq & C \gamma^{n} e^{\omega t} \quad(t \geqq 0) .
\end{aligned}
$$

Then, since $S *\left(P_{1} * S\right)^{* n}$ is strongly continuous in $t \geqq 0$ (see again [6], Lemma VIII. 1.21) and (5.7) guarantees strong uniform convergence on compacts of $t \geqq 0, S_{1}=\left(P+P_{1}\right)^{-1}$ is itself strongly continuous in $t \geqq 0$ and

$$
\left|S_{1}(t)\right| \leqq C(1-\gamma)^{-1} e^{\omega t} \quad(t \geqq 0) .
$$

6. The abstract parabolic case. We say that $P \in \mathscr{D}_{0}^{\prime-1}$ is abstract parabolic if $S=P^{-1}$ coincides in $t>0$ with $C^{\infty}(E ; X)$-valued function (in symbols, $\left.P \in\left(C^{\infty}\right)^{-1}\right)$. Abstract parabolic distributions of the form $P=\delta^{\prime} \otimes I-\delta \otimes A$ were characterized by Pazy [20] in the case where $A$ is a semigroup generator and by Barbu [1] when $P \in$ $\mathscr{D}_{0}^{\prime-1}$. Our proof of Theorem 5.1 is close to that of Barbu.

THeOREM 6.1. Let $P \in \mathscr{E}^{\prime}$. Then $P$ is abstract parabolic if and only if for every $\alpha>0$ there exist $\beta=\beta(\alpha), \omega=\omega(\alpha)>0$ such that $\rho(P)$ contains the region

$$
\Omega(\alpha, \beta, \omega)=\{\lambda ; \operatorname{Re} \lambda \geqq \min (\beta-\alpha \log |\lambda|, \omega)\}
$$




$$
\left|\mathfrak{P}(\lambda)^{-1}\right|_{(E ; X)} \leqq C(1+|\lambda|)^{m} \quad(\lambda \in \Omega(\alpha, \beta, \omega))
$$

where $C-$ but not $m-$ may depend on $\alpha$.

Proof. By virtue of Lemma 2.4, $P \in \mathscr{S}_{0}^{\prime-1}$. We use now the expression (2.14) for $Y_{p} * S$, the $p$ th antiderivative of $S$ with, say, $p>m+1$. Then the contour of integration in (2.14) can be deformed to $\Sigma(\alpha)$, boundary of $\Omega(\alpha, \beta(\alpha)+1, \omega+1)$ for $\alpha$ arbitrary. Now, in the last integral the integrand is $O\left(|\lambda|^{-\alpha t-p+m}\right)$ so that, if $t>(m+1) / \alpha$ we can differentiate $p$ times under the integral sign and finally obtain

$$
S(t)=\frac{1}{2 \pi i} \int_{\Sigma(\alpha)} e^{\lambda t} \mathfrak{P}(\lambda)^{-1} d \lambda
$$

Now, it is obvious that (6.2) holds for $t>(m+1) / \alpha$ where $\alpha$ is arbitrary, so that $P \in\left(C^{\infty}\right)^{-1}$ as claimed.

We prove now the converse. Assume that $S=P^{-1}$ is $C^{\infty}$ for $t>0$, where $P \in \mathscr{E}^{\prime}$. Let $\operatorname{supp}(P) \subseteq[0, b]$ and, given $\alpha>0$ choose $0<a<$ $1 / 2$ and an integer $p$ in such a way that

$$
p(1+b)^{-1} \geqq \alpha, \quad p a(1+b)^{-1} \leqq 1 / 2
$$

Let now $\varphi \in \mathscr{H}_{a} . \quad$ As in Theorem 2.5 we have

$$
P * \varphi S=\delta \otimes I-\Phi, \quad \varphi S * P=\delta \otimes I-\Psi
$$

but now $\Phi=P *(1-\varphi) S=\delta \otimes I-P * \varphi S$ is a $C^{\infty}(E ; E)$-valued function with support in $a \leqq t \leqq 2 a+b$ whereas $\Psi$ is an $(X ; X)$-valued function with the same properties. After repeated integration by parts we have

$$
\mathscr{L} \Phi(\lambda)=\lambda^{-p} \int_{0}^{\infty} e^{-\lambda t} \Phi^{(p)}(t) d t
$$

hence

$$
\begin{gathered}
|(\mathscr{L} \Phi)(\lambda)| \leqq C|\lambda|^{-p} \quad(\operatorname{Re} \lambda \geqq 0) \\
|(\mathscr{L} \Phi)(\lambda)| \leqq C|\lambda|^{-p} e^{-(2 a+b) \operatorname{Re} \lambda} \quad(\operatorname{Re} \lambda<0) .
\end{gathered}
$$

Let now $\gamma<1$. A short computation shows that

$$
|\mathscr{L} \Phi(\lambda)|_{(E ; E)} \leqq \gamma \quad \text { for } \quad \lambda \in \Omega(\alpha, \beta, \omega)
$$

if $\beta=(2 a+b)^{-1} \log C \gamma^{-1}, \omega=\left(C \gamma^{-1}\right)^{1 / p}$. Operating in the same way with $\Psi$ and modifying if necessary $\beta$, $\omega$ we obtain 


$$
|\mathscr{L} \psi(\lambda)|_{(X ; X)} \leqq \gamma \quad \text { for } \quad \lambda \in \Omega(\alpha, \beta, \omega) .
$$

Observe next that, for some integer $q$

$$
\begin{array}{ll}
|\mathscr{L}(\varphi S)(\lambda)|_{(E ; X)} \leqq C|\lambda|^{q} & (\operatorname{Re} \lambda \geqq 0) \\
|\mathscr{L}(\varphi S)(\lambda)|_{(E ; X)} \leqq C|\lambda|^{a} e^{-2 a \operatorname{Re} \lambda} & (\operatorname{Re} \lambda<0)
\end{array}
$$

(here and in the following inequalities $C$ is not necessarily the same constant). According to (6.3) we have $2 \alpha a \leqq 2 a p(1+b)^{-1} \leqq 1$ so that we obtain from (6.8)

$$
\begin{aligned}
|\mathscr{L}(\varphi S)(\lambda)|_{(E ; X)} & \leqq C|\lambda|^{q+2 \alpha a} \\
& \leqq C|\lambda|^{q+1} \quad(\lambda \in \Omega(\alpha, \beta, \omega) .
\end{aligned}
$$

Combining now (6.6) and (6.7) as in Theorem 2.5 we see that $\mathfrak{P}(\lambda)^{-1}=$ $\mathscr{L}(\varphi S)(I+\mathscr{L} \Phi(\lambda))^{-1}$ in $\Omega(\alpha, \beta, \omega)$ and the result follows from (6.9).

REMARK 6.2. Note that we obtain as a by-product of Theorem 6.1 (see Barbu [1]) that if $P \in\left(C^{\infty}\right)^{-1}$ then $S$ grows exponentially at infinity; more precisely, (6.2) implies

$$
|S(t)|_{(E ; X)} \leqq C_{\epsilon} e^{\omega t} \quad(t \geqq \epsilon)
$$

for any $\epsilon>0$.

REMARK 6.3. We don't know of a characterization of $P \in\left(C^{\infty}\right)^{-1}$ for $P \in \mathscr{S}^{\prime}$ not having compact support. The conditions in Theorem 6.1 are certainly sufficient even if $P \notin \mathscr{E}_{0}^{\prime}$ but they are no longer necessary; if $P \in \mathscr{D}_{0}^{\prime}$ and these conditions are verified for $\varphi P$ for any $\varphi \in \mathscr{H}$ then it is easy to see (using Lemma 2.2 in the style of Theorem 2.7) that $P \in\left(C^{\infty}\right)^{-1}$. We do not know whether this is a necessary condition.

It seems natural to ask whether the condition that $P \in \mathscr{E}^{\prime} \cap\left(C^{\infty}\right)^{-1}$ implies any smoothness properties of $P$ itself. Somewhat surprisingly, the answer to this question turns out to be affirmative when $\operatorname{dim} E<\infty$ but negative in general.

The following result is an adaptation of a theorem in Ehrenpreis [7]. We follow closely the proof of Hörmander [16]

Theorem 6.4. Let $\operatorname{dim} E<\infty$. Assume that $P \in \mathscr{E}^{\prime} \cap\left(C^{\infty}\right)^{-1}$. Then $P$ coincides with a $C^{\infty}$ function with values in $(X ; E)$ for $t>0$.

Proof. Note (see the remarks following (3.11)) that $\operatorname{dim} X=\operatorname{dim} E$, 
so we may take $X=E$; we assume coordinates have been introduced in $E$ and work with matrices instead of operators. Let $P=0$ for $t \geqq a$ and choose $\varphi \in \mathscr{H}_{a}$. We take the Laplace transform of the first equation (6.4), pre-multiply both sides by $\mathfrak{B}(\lambda)^{-1}$ and take determinants. We obtain, for every $\alpha>0$

$$
\begin{gathered}
f(\lambda)=\operatorname{det} \mathscr{L}(\varphi S)(\lambda)=\operatorname{det}(I+\mathscr{L}(\Phi)(\lambda)) \operatorname{det} \mathfrak{B}(\lambda)^{-1} \\
(\lambda \in \Omega(\alpha, \beta(\alpha), \omega(\alpha))
\end{gathered}
$$

so that, in view of (6.6), $f(\lambda)$ does not have zeros in any of the regions $\Omega(\alpha, \beta(\alpha), \omega(\alpha))$. This is easily seen to imply that, if $\left\{\lambda_{n}\right\}$ are the zeros of $f$ (say, arranged in order of increasing modulus),

$$
\operatorname{Re} \lambda_{n} / \log \left|\lambda_{n}\right| \rightarrow-\infty \quad \text { as } \quad n \rightarrow \infty
$$

We take now inverses in (6.10),

$$
1 / f(\lambda)=\operatorname{det} \mathfrak{B}(\lambda) \operatorname{det}(I+\mathscr{L}(\Phi)(\lambda))^{-1} .
$$

Since $|\mathfrak{P}(\lambda)|<C(1+|\lambda|)^{m}$ for $\operatorname{Re} \lambda>0$, a similar inequality must be satisfied by each of the entries of $\mathfrak{P}(\lambda)$; therefore $\mid \operatorname{det} \mathfrak{B}($ i $\eta) \mid \leqq$ $C(1+|\eta|)^{m n}(n=\operatorname{dim} E)$ and then it follows from (6.12) that

$$
|f(i \eta)| \geqq C(1+|\eta|)^{-m} \quad(-\infty<\eta<\infty) .
$$

Finally, note that $f(\lambda)$ is the Laplace transform of a distribution with singular support equal to $\{0\}$ (the "convolution determinant" of $\varphi S$ ). It has been proved by Hormander [16] that this, (6.11) and (6.13) imply that $1 / f$ satisfies the conditions set up for $\mathfrak{P}^{-1}$ in the proof of Theorem 5.1, that is, for all $\alpha>0|1 / f(\lambda)| \leqq C(1+|\lambda|)^{m}(\lambda \in \Omega(\alpha, \beta(\alpha), \omega(\alpha))$. Observe next that, since sing $\operatorname{supp}(\varphi S)=\{0\},|\mathscr{L}(\varphi S)(\lambda)|$, thus each of the entries of the matrix $\varphi S$, must obey a family of inequalities of the same type of (6.11). It follows then that the entries of $\mathscr{L}(\varphi S)(\lambda)^{-1}$-thus $\left|\mathscr{L}(\varphi S)(\lambda)^{-1}\right|$ - must satisfy the assumptions of Theorem 5.1 ; accordingly, $(\varphi S)^{-1} \in C^{\infty}$. But, by virtue of Lemma $(\varphi S)^{-1}=P$ in $t \leqq a$, which proves the result.

REMARK 6.5. Theorem 6.4 becomes false when $\operatorname{dim} E=\infty$. Examples showing this can be given by "interchanging a semigroup with its generator" as follows. Let $S$ be a semigroup with no tendency towards smoothness - say, a group with unbounded generator $A$. Set $E=$ $D(A)$ with the graph norm, $X=E$. Then, if $P=S$, 


$$
P^{-1}=\delta^{\prime} \otimes I-\delta \otimes A
$$

which is clearly $C^{\infty}$ - in fact, zero - in $t>0$. We can obtain an even worse $P$ by replacing $S$ by a regular group distribution with unbounded generator or by using $P=(d / d s)^{m} S$, in which case $P^{-1}=$ $Y_{m-1} \otimes I-Y_{m} \otimes A$.

7. The strong Cauchy problem. We assume in this section that $X \rightarrow E$, that is, $X$ is a dense subspace of $E$ and the injection map $i: X \rightarrow E$ is continuous. Let $P \in \mathscr{Y}_{0}^{\prime}((X ; E))$ and write

$$
P=\mu^{(m)}
$$

where $\mu$ is a measure with values in $(X ; E)$ and $m$ is the least integer for which a representation of the type of $(7.1)$ is possible. Denote by $U_{0}$ the space of all $X$-valued functions $u_{0}(t)$ defined in $t \leqq 0, m$ times continuously differentiable and with compact support.

We study the following version of the Cauchy problem: given $u_{0} \in \mathcal{U}_{0}$, to find an $E$-valued continuous function $u(t)$ defined in $t \geqq 0$ such that $u(\cdot)$ (extended to be zero in $t<0$ ) defines a distribution in $\mathscr{D}_{0}^{\prime}((X ; E))$ and

$$
P *\left(u+u_{0}\right)=0 \quad(t \geqq 0) .
$$

The definition of solution can be made more stringent in several ways (for instance, we may require $u$ to be an $m$ times continuously differentiable function with values in $X$, so that $u$ is a "true" solution of (7.2)). The Cauchy problem in this sense appears in connection with systems whose behavior is described by "hereditary" equations, for instance differencedifferential or integro-differential equations, integration being performed with respect to time. The "initial function" $u_{0}$ is the history of the system up to $t=0$; at that time, the equation (7.2) takes over and determines the future evolution of the system. We can also consider the inhomogeneous equation

$$
P *\left(u_{0}+u\right)=f \quad(t \geqq 0)
$$

where $f$ is, say, an $E$-valued continuous function in $t \geqq 0$ and vanishes in $t<0$ and is interpreted as an external influence acting upon the system. An important particular case of (7.3) is that where $u_{0}=0$ for $t \leqq 0$; here the system is dormant until $t=0$, at which time begins to be excited by $f$. In this case, (7.3) reduces to

$$
P * u=f
$$


If $P \in \mathscr{D}_{0}^{\prime-1}$, the formal solution of (7.3) is

$$
u=S *\left(f-\left\{P * u_{0}\right\}_{0}\right)
$$

where $S=P^{-1}$ and $\{\cdot\}_{0}$ indicates truncation at zero; more precisely

$$
\left\{P * u_{0}\right\}_{0}=P * u_{0}-w
$$

where $w .(t)=\left(P * u_{0}\right)(t)$ for $t<0, w(t)=0$ for $t \geqq 0$ (note that $P * u_{0}$ is a continuous $X$-valued function for $t \leqq 0)$. Clearly, 7.5 is the only distribution satisfying (7.3).

Let $\|\cdot\|$ be a semi-norm in $U_{0}$. It seems natural to say that the Cauchy problem for (7.3) is well posed (with respect to $\|\cdot\|$ ) if the solution $u$ of (7.3) (given by (7.5)) is $E$-strongly continuous in $t \geqq 0$ for any $u_{0} \in U_{0}$ and $f$ strongly continuous and

$$
|u(t)| \leqq \eta(t)\left(\left\|u_{0}(\cdot)\right\|+\int_{0}^{t}|f(s)| d s\right) \quad(t \geqq 0)
$$

where $\eta$ is a continuous function independent of $u_{0}, f$. This definition can clearly be modified in many ways. We present now four examples to illustrate the form of (7.5), (7.6) in several particular cases and to deduce conditions that assure that (7.6) will hold.

Example 1. Let $P=\delta^{\prime} \otimes I-\delta \otimes A$ with $A$ closed and densely defined, $X=D(A)$ with the graph norm $|u|_{A}=|u|+|A u|$. If $u_{0} \in U_{0}$ we define $\left\|u_{0}\right\|=\left|u_{0}(0)\right|$. Since $\left\{P * u_{0}\right\}_{0}=-\delta \otimes u_{0}(0),(7.5)$ reduces to

$$
u(t)=S(t) u_{0}(0)+\int_{0}^{t} S(t-s) f(s) d s
$$

if $S$ is a strongly continuous $(E ; E)$-valued function in $t \geqq 0$. But then $S$ must be a strongly continuous semigroup, thus $|S(t)| \leqq C e^{\omega t}$ and (7.6) is satisfied with $\eta(t)=C e^{\omega t}$.

Example 2. Let $P=\delta^{\prime \prime} \otimes I-\delta^{\prime} \otimes B-\delta \otimes A$, where $A$ and $B$ are closed operators such that $D(A) \cap D(B)=X$ is dense in $E$; we use in $X$ the norm $|u|+|A u|+|B u|$. Define $\left\|u_{0}(0)\right\|=\left|u_{0}(0)\right|+\left|u_{0}^{\prime}(0)\right|$. Since $\left\{P * u_{0}\right\}_{0}=-\delta^{\prime} \otimes u_{0}(0)-\delta \otimes\left(u_{0}^{\prime}(0)-B u_{0}(0)\right)$, we have

$$
\begin{aligned}
u(t)= & \left(S^{\prime}(t)-S(t) B\right) u_{0}(0)+S(t) u_{0}^{\prime}(0) \\
& +\int_{0}^{t} S(t-s) f(s) d s
\end{aligned}
$$


and (7.6) will be satisfied if $S(t), S^{\prime}(t)$ and $S(t) B$ are strongly continuous $(E ; E)$-valued functions in $t \geqq 0$. In this case, however, it is not necessarily true that $\eta(t) \leqq C e^{\omega t}$ for some $C, \omega$ (see [11]).

EXAMPLE 3. We consider the difference-differential operator $P=$ $\delta^{\prime}(\hat{t}) \otimes I-\delta(\hat{t}) \otimes A-\delta(\hat{t}-1) \otimes B$ where $A$ and $B$ satisfy the same conditions as in Example 2. Now we define $\left\|u_{0}\right\|=\sup _{-1 \leqq t \leqq 0}\left|u_{0}(t)\right|$ for $u_{0} \in \mathcal{U}_{0}$. We have $\left\{P * u_{0}\right\}_{0}=-\delta \otimes u_{0}(0)-B u_{0}(t-1)$ and, assuming that $S$ is strongly continuous in $t \geqq 0$.

$$
u(t)=S(t) u_{0}(0)+\int_{0}^{1} S(t-s) B u_{0}(s-1) d s
$$

$$
+\int_{0}^{t} S(t-s) f(s) d s
$$

We obtain an inequality of the type of (7.6) if, in addition to strong continuity of $S$ we assume

$$
\int_{t}^{t+1}|S(s) B u|_{E} d s \leqq \nu(t)|u|_{E} \quad(t \geqq 0, u \in X)
$$

for some locally bounded function $\nu(t)>0$.

Example 4. Let $P=\delta^{\prime \prime} \otimes I-\delta \otimes A-\gamma \otimes A$ where $\gamma$ is a bounded scalar function in $t \geqq 0, A$ is a closed, densely defined operator and $X=D(A)$ with the graph norm. Let $u_{0} \in \mathcal{U}_{0}$ and define

$$
\left\|u_{0}\right\|=\left|u_{0}(0)\right|+\left|u_{0}^{\prime}(0)\right|+\int_{-\infty}^{0}\left|u_{0}(s)\right| d s
$$

Now

$$
\left\{P * u_{0}\right\}_{0}=-\delta \otimes u_{0}^{\prime}(0)-\delta^{\prime} \otimes u_{0}(0)-\int_{-\infty}^{t} \gamma(t-s) u_{0}(s) d s
$$

so that

$$
\begin{aligned}
u(t)= & S(t) u_{0}^{\prime}(0)+S^{\prime}(t) u_{0}(0) \\
& +\int_{0}^{t} S(t-s)\left\{\int_{-\infty}^{s} \gamma(s-r) u_{0}(r) d r+f(s)\right\} d s .
\end{aligned}
$$

Assuming that $S, S^{\prime}$ are strongly continuous $(E ; E)$-valued functions in 
$t \geqq 0$ we obtain easily (7.6). Under the same hypotheses, but this time assuming $\gamma$ summable, we obtain (7.6) for the semi-norm

$$
\left\|u_{0}\right\|=\left|u_{0}(0)\right|+\left|u_{0}^{\prime}(0)\right|+\sup _{-\infty \leqq t \leqq 0}\left|u_{0}(t)\right| .
$$

We note that if we only require (7.6) for the case $u_{0}=0$ (systems which are at rest until $t=0)$, it suffices in all cases to require that $S$ be a strongly continuous $(E ; E)$-valued function for $t \geqq 0$.

8. Two applications. (i) We examine the differencedifferential equation

$$
u^{\prime}(t)=A u(t)+B u(t-1)+f(t) \quad(t \geqq 0)
$$

in the spirit of $\$ 7$, especially Example 3 . Here $A$ is the infinitesimal generator of a strongly continuous semigroup $S(t) ; t \geqq 0$ and $B$ is a closed operator with $D(B) \supseteq D(A)$. We assume that $B$ is "subordinated" to $A$ in the following sense: there exist two continuous functions $\alpha(t), \beta(t)$ defined in $t>0$ such that

$$
\begin{aligned}
& \text { (a) }|B S(t) u| \leqq \alpha(t)|u| \quad(t>0, u \in D(A)) \\
& \text { (b) }|S(t) B u| \leqq \beta(t)|u| \quad(t>0, u \in D(B)) \\
& \text { (c) } \int_{0}^{1} \alpha(t) d t<\infty \quad \int_{0}^{1} \beta(t) d t<\infty .
\end{aligned}
$$

We begin by showing that (a) implies that

$$
P=\delta^{\prime}(\hat{t}) \otimes I-\delta(\hat{t}) \otimes A-\delta(\hat{t}-1) \otimes B
$$

belongs to $\mathscr{D}_{0}^{\prime-1}((X ; E))$, where $X=D(A)$ endowed with the graph norm. We do this by pertubation of $P_{0}=\delta^{\prime}(\hat{t}) \otimes I-\delta(\hat{t}) \otimes A$ by $P_{1}=\delta(\hat{t}-1) \otimes B$. Observe first that (8.2) implies that for every $t>0$ the operator $B S(t)$ admits a bounded extension $\overline{B S(t)} \in(E ; E)$ such that

$$
\overline{|B S(t)|} \leqq \alpha(t) \quad(t>0)
$$

Moreover, $\overline{B S(\cdot)}$ is strongly continuous in $t>0$; to see this, in view of (8.5) it is enough to show that $B S(\cdot) u$ is continuous for $u$ in a dense subset of $E$. But, if $u \in D(A), B S(t) u=B R(\lambda ; A) S(t)(\lambda I-A) u$ and $B R(\lambda ; A)$ is bounded by virtue of the closed graph theorem.

Observing now that $\left(P_{1} * S u\right)(t)=B S(t-1) u$ (we set $S(t)=0$ for $t<0)$ for $u \in D(A)$ it is not difficult to deduce that 


$$
\left(P_{1} * S\right)(\hat{t})=\overline{B S(\hat{t}-1)}
$$

We look now at the perturbation series

$$
\begin{aligned}
S_{1}(\hat{t})= & S(\hat{t})+S(\hat{t}) * \overline{B S(\hat{t}-1)} \\
& +S(\hat{t}) * B S(\hat{t}-1) * \overline{B S(\hat{t}-1)}+\cdots
\end{aligned}
$$

Convergence of (8.7) is clear, as the $n^{t h}$ term vanishes for $t \leqq n-1$; according to Theorem 5.1,

$$
S_{1}=P^{-1}
$$

We note that, again by virtue of Lemma VIII. 1.21 in [6] all the terms in (8.7) are $(E ; E)$-valued strongly continuous functions in $t \geqq 0$ so that the same is true of $S_{1}$. We estimate now (8.7) using Remark 5.3. Clearly, an estimate of the form (5.8) will hold if we can prove that

$$
\int_{0}^{\infty} e^{-\omega t} \alpha(t) d t<\infty
$$

for some $\omega>0$; in view of the first condition (c), we only have to prove that $\alpha(t)$ grows exponentially at infinity. But this follows easily from the inequality $|B S(t) u| \leqq \alpha\left(t^{\prime}\right)\left|S\left(t-t^{\prime}\right) u\right|\left(u \in B, 0 \leqq t^{\prime} \leqq t\right)$. Accordingly, (8.8) holds and

$$
\left|S_{1}(t)\right| \leqq C e^{\omega t} \quad(t \geqq 0)
$$

for some $C, \omega$.

We rewrite now (8.7) using (8.6) and the associativity of the convolution product,

$$
S_{1}=S+\left(S * P_{1}\right) * S+\left(S * P_{1}\right)^{* 2} * S+\cdots
$$

Now, it is immediate that, if $u \in X\left(S * P_{1} u\right)(t)=S(t-1) B u$. Using arguments similar to the ones preceding and following (8.5) we see that $S(t) B$ can be extended to an $(E ; E)$-valued strongly continuous function satisfying

$$
\overline{|S(t) B|} \leqq \beta(t)
$$

An approximation argument then shows 


$$
\begin{aligned}
S_{1}(\hat{t})= & S(\hat{t})+\overline{S(\hat{t}) B} * S(\hat{t}-1) \\
& +\overline{S(\hat{t}) B} * \overline{S(\hat{t}-1) B} * S(\hat{t}-1)+\cdots
\end{aligned}
$$

accordingly, if $u \in D(B)$,

$$
\begin{aligned}
S_{1}(\hat{t}) B u= & \overline{S(\hat{t}) B u}+\overline{S(\hat{t}) B} * \overline{S(\hat{t}-1) B u} \\
& +\overline{S(\hat{t}) B} * \overline{S(\hat{t}-1) B} * \overline{S(\hat{t}-1) B u}
\end{aligned}
$$

so that $\left|S_{1}(\hat{t}) B u\right|$ can be estimated by

$$
\left(\beta(\hat{t})+\beta(\hat{t}) * \beta(\hat{t}-1)+\beta(\hat{t}) * \beta(\hat{t}-1)^{* 2}+\cdots\right)|u| \leqq \beta_{1}(\hat{t})|u| .
$$

Now, it is easy to show, as for $\alpha$, that $\beta$ satisfies (8.8) and then, on the basis of Young's inequality, that

$$
\int_{0}^{\infty} e^{-\omega t}\left|\beta_{1}(t)\right| d t<\infty
$$

for some $\omega>0$. This is easily seen to imply

$$
\int_{t}^{t+1}|S(s) B u| d s \leqq C e^{\omega t}|u|_{E} \quad(t \geqq 0)
$$

so that (see again Example 4, §7) we have, in view of (7.10) and (8.9)

$$
|u(t)|<C e^{\omega t}\left(\sup _{-1 \leqq t \leqq 0}\left|u_{0}(t)\right|+\int_{0}^{t}|f(s)| d s\right) \quad(t \geqq 0)
$$

for suitable constants $C, \omega$.

We consider now a concrete partial difference-differential operator where the preceding considerations apply. Let $E$ be the space of all continuous functions $u$ defined and continuous in $-\infty<x<\infty$ and such that $\lim _{|x| \rightarrow \infty} u(x)=0$ endowed with its usual supremum norm. The operator $A$ is defined by

$$
(A u)(x)=u^{\prime \prime}(x)
$$

$D(A)$ the set of all twice continuously differentiable $u$ for which $u, u^{\prime}, u^{\prime \prime} \in E$. As for $B$,

$$
(B u)(x)=b(x) u^{\prime}(x)
$$

$D(B)$ the set of all continuously differentiable $u$ for which $u, b u^{\prime} \in$ 
E. We assume $b$ continuous and bounded, so that clearly $D(A) \subseteq$ $D(B)$. If moreover, $b$ is continuously differentiable $B$ is closed. As for $A$, it is well known that it generates a strongly continuous semigroup $S(\cdot)$ in $E$ given by the formula

$$
(S(t) u)(x)=\int_{-\infty}^{\infty} K(t, x-y) u(y) d y
$$

where $K$ is the Weierstrass kernel $(4 \pi t)^{-1 / 2} \exp \left(x^{2} / 4 t\right)$. Some routine computations show that (a) holds with $\alpha(t)=M(\pi t)^{-1 / 2}$ where $M=$ $\sup |b(x)|$. Assume in addition that $\left|b^{\prime}(x)\right|=O(|x|)$ as $|x| \rightarrow \infty$; then (b) holds with $\beta$ similar to $\alpha$. Thus the results apply to the partial difference-differential equation

$$
\frac{\partial u}{\partial t}(x, t)=\frac{\partial^{2} u}{\partial x^{2}}(x, t)+b(x) \frac{\partial u}{\partial x}(x, t-1) \quad(t \geqq 0)
$$

(i) Gurtin and Pipkin [14] propose a generalized heat equation that, in the case of one space variable can be written, after linearization,

$$
\begin{aligned}
& c \frac{\partial^{2} u}{\partial t^{2}}(x, t)+\beta(0) \frac{\partial u}{\partial t}(x, t)+\int_{0}^{\infty} \beta^{\prime}(s) \frac{\partial u}{\partial t}(x, t-s) d s \\
& \quad=a(0) \frac{\partial^{2} u}{\partial x^{2}}(x, t)+\int_{0}^{\infty} a^{\prime}(s) \frac{\partial^{2} u}{\partial x^{2}}(x, t-s) d s+f(x, t) .
\end{aligned}
$$

When $\beta=0$ this equation also arises in the theory of viscoelasticity (see [14]). For the sake of simplicity we shall only consider this case here, although our methods apply equally well to the complete equation (8.13). We shall also suppose, to simplify some formulas, that $c=$ $a(0)=1$. We consider then (see $\S 7$, Example 4)

$$
P=\delta^{\prime \prime} \otimes I-\delta \otimes A-a^{\prime} \otimes A \text {. }
$$

The space $E$ and the operator $A$ are defined in the same way as in (i). Again we write $P=P_{0}+P_{1}$ with $P_{0}=\delta^{\prime \prime} \otimes I-\delta \otimes A, P_{1}=a^{\prime} \otimes A$ and we assume $a$ to be twice continuously differentiable and $a^{\prime \prime} \in$ $L^{1}(0, \infty)$. It is well known (D'Alembert's formula) that $P_{0} \in$ $\mathscr{D}_{0}^{\prime-1}((E ; X))$ and that $S=P_{0}^{-1}$ is given by

$$
S(t) u(x)=\frac{1}{2} \int_{x-t}^{x+t} u(y) d y .
$$

Note that $S$ is a continuously differentiable $(E ; E)$-valued function and 


$$
S^{\prime}(t) u(x)=(1 / 2)(u(x+t)+u(x-t))
$$

which is itself $(E ; E)$-valued and strongly continuous. To apply Theorem 5.1 we compute $P_{1} * S$; if $u \in E$ is continuously differentiable we have

$$
\begin{aligned}
\left(\left(P_{1} * S u\right)(t)\right)(x) & =\int_{0}^{t} a^{\prime}(t-s)\left\{\frac{\partial^{2}}{\partial x^{2}} \cdot \frac{1}{2} \int_{x-s}^{x+s} u(y) d y\right\} d s \\
& =\frac{1}{2} \int_{0}^{t} a^{\prime}(t-s)\left(u^{\prime}(x+s)-u^{\prime}(x-s)\right) d s \\
& =\frac{1}{2} a^{\prime}(0)(u(x+t)+u(x-t))-a^{\prime}(t) u(x) \\
& +\frac{1}{2} \int_{0}^{t} a^{\prime \prime}(t-s)(u(x+s)+u(x-s)) d s
\end{aligned}
$$

Taking now into account that $a^{\prime \prime} \in L^{1}$ and that, accordingly, $a^{\prime}(t) \rightarrow 0$ as $t \rightarrow \infty$ we see that $\left(P_{1} * S\right)$ is a strongly continuous function with values in $(E ; E)$ and

$$
\left|\left(P_{1} * S\right)(t)\right| \leqq C \quad(t \geqq 0)
$$

so that we deduce from Theorem 5.1 and from Remark 5.3 that $P \in \mathscr{D}_{0}^{\prime-1}((E ; X))$ and that $S_{1}=P_{1}^{-1}$ is a strongly continuous function given by

$$
S_{1}=S+S *\left(P_{1} * S\right)+S *\left(P_{1} * S\right)^{* 2}+\cdots
$$

Now, $S$ is continuously differentiable (see (8.14) and following comments) and it is easy to see that (8.18) can be differentiated term by term. Hence $S_{1}$ itself is continuously differentiable and $S_{1}^{\prime}$, which is itself an $(E ; E)$-valued strongly continuous function, is given by

$$
S_{1}^{\prime}=S^{\prime}+S^{\prime} *\left(P_{1} * S\right)+S^{\prime} *\left(P_{1} * S\right)^{* 2}+\cdots
$$

Estimating the series (8.18) and (8.19) in the style of $\$ 5$ we see that, given $\omega>0$ there exists $C=C_{\omega}$ with

$$
\left|S_{1}(t)\right| \leqq C e^{\omega t}, \quad\left|S_{1}^{\prime}(t)\right| \leqq C e^{\omega t}
$$

so that, if $u(\cdot)$ is the solution of (8.13) corresponding to a $u_{0} \in \mathcal{U}_{0}$ (see $\S 7$, Example 4) we have

$$
|u(t)| \leqq C e^{\omega t}\left(\left|u_{0}(0)\right|+\left|u_{0}^{\prime}(0)\right|+\int_{-\infty}^{0}\left|u_{0}(s)\right| d s+\int_{0}^{t}|f(s)| d s\right) .
$$


If we assume $a^{\prime}$ summable, then there is an inequality of the same type but using the semi-norm (7.13) for the initial function $u_{0}$.

\section{REFERENCES}

1. V. Barbu, Les semi-groupes distributions différentiables, C. R. Acad. Sci. Paris, 267 (1968), 875-878.

2. J. Chazarain, Problèmes de Cauchy au sens des distributions vectorielles et applications, C. R. Acad. Sci. Paris, 266 (1968), 10-13.

3. J. Chazarain, Problèmes de Cauchy abstraits et applications a quelques problèmes mixtes, J. Functional Analysis, 7 (1969), 386-446.

4. G. Da Prato-U. Mosco, Semigruppi distribuzioni analitici, Ann. Scuola Norm. Sup. di Pisa, XIX (1965), 367-396.

5. G. Da Prato and U. Mosco, Regolarizzazione dei semigruppi distribuzioni analitici, Annali della Scuola Normale Superiore di Pisa, XIX (1965), 563-576.

6. N. Dunford and J. T. Schwartz, Linear operators, Part 1, Interscience, N. Y. 1957.

7. L. Ehrenpreis, Solution of some problems of division IV: Invertible and Elliptic Operators, Amer.

J. Math., 32 (1960), 522-588.

8. H. O. Fattorini, Ordinary differential equations in linear topological spaces I, J. Differential Equations, 5 (1969), 72-105.

9. - Ordinary differential equations in linear topological spaces II, J. Differential Equations, 6 (1969), 50-70.

10. On a class of differential equations for vector-valued distributions, Pacific J. Math., 32 (1970), 79-104.

11. Extension and behavior at infinity of solutions of certain operational differential equations, Pacific J. Math., 33 (1970), 583-615.

12. C. Foias, Remarques sur les semi-groupes distributions d'opérateurs normaux, Port. Math., 19 (1960), 227-242.

13. D. Fujiwara, A characterization of exponential distribution semi-groups, J. Math. Soc. Japan, 18 (1966), 267-274.

14. M. A. Gurtin and A. C. Pipkin, A general theory of heat conduction with finite wave speeds, Archive Rat. Mech. Anal., 31 (1968), 113-126.

15. E. Hille and R. S. Phillips, Functional analysis and semı-groups, Amer. Math. Soc., Providence, 1957.

16. L. Hörmander, Hypoelliptic convolution equations, Math. Scand., 9 (1961), 178-184.

17. J. L. Lions, Problèmes aux limites en théorie des distributions, Acta Math., 94 (1955), 13-153.

18. - Les semi-groupes distributions, Port. Math., 19 (1960), 141-164.

19. J. L. Lions and E. Magenes, Problèmes aux limites non homogènes et applications, vol. 3, Dunod, Paris, 1971.

20. A. Pazy, On the differentiability and compactness of semi-groups of linear operators, J. Math. Mech., 17 (1968), 1131-1142.

21. L. Schwartz, Théorie des distributions, Hermann, Paris, 1966.

22. - Théorie des distributions a valeurs vectorielles, I, Ann. Inst. Fourier, VII (1957), 1-141.

23. - Théorie des distributions a valeurs vectorielles II, Ann. Inst. Fourier, VIII (1958), 1-209.

24. M. Sova, Problème de Cauchy pour équations hyperboliques à coefficients constants non-bornés, Ann. Scuola Norm. Sup. Pisa, XXII (1968), 67-100.

25. K. Yosida, On the differentiability of semi-groups of linear operators, Proc. Japan Acad., 34 (1958), 337-340.

Received May 8, 1975 and in revised form May 3, 1976.

University of CAlifornia, Los ANgeles 



\section{PACIFIC JOURNAL OF MATHEMATICS}

\section{EDITORS}

RICHARI) ARENS (Managing Editor)

University of California

Los Angeles, CA 90024

R. A. Beaumiont

University of Washington

Seattle, WA 98105

\section{J. DugunduI}

Department of Mathematics University of Southern California Los Angeles, CA 90007

D. Gilbarg and J. Milgram Stanford University

Stanford, CA 94305

\section{ASSOCIATE EDITORS}

E. F. BECKENBACH
B. H. NEUMANN

F. Wolf

K. YoshidA

\section{SUPPORTING INSTITUTIONS}

UNIVERSITY OF BRITISH COLUMBIA CALIFORNIA INSTITUTE OF TECHNOLOGY

UNIVERSITY OF CALIFORNIA

MONTANA STATE UNIVERSITY

UNIVERSITY OF NEVADA

NEW MEXICO STATE UNIVERSITY

OREGON STATE UNIVERSITY

UNIVERSITY OF OREGON

OSAKA UNIVERSITY

\author{
UNIVERSITY OF SOUTHERN CALIFORNIA \\ STANFORD UNIVERSITY \\ UNIVERSITY OF HAWAII \\ UNIVERSITY OF TOKYO \\ UNIVERSITY OF UTAH \\ WASHINGTON STATE UNIVERSITY \\ UNIVERSITY OF WASHINGTON \\ AMERICAN MATHEMATICAL SOCIETY
}

The Supporting Institutions listed above contribute to the cost of publication of this Journal, but they are not owners or publishers and have no responsibility for its contents or policies.

Mathematical papers intended for publication in the Pacific Journal of Mathematics should be in typed form or offset-reproduced (not dittoed), double spaced with large margins. Underline Greek letters in red, German in green, and script in blue. The first $p: 1<$ graph or two must be capable of being used separately as a synopsis of the entire paper. Items of the biblography should not be cited there unless absolutely necessary, in which case they must he identified by author and Journal, rather than by item number. Manuscripts, in duplicate, may be sent to any one of the four editors. Please classify according to the scheme of Math. Reviews, Index to Vol. 39. All other communications should be addressed to the managing editor, or Elaine Barth, University of California, Los Angeles, California, 90024.

100 reprints are provided free for each article, only if page charges have been substantially paid. Additional copies may be obtained at cost in multiples of 50 .

The Pacific Journal of Mathematics is issued monthly as of January 1966. Regular subscription rate: $\$ 72.00$ a year (6 Vols., 12 issues). Special rate: $\$ 36.00$ a year to individual members of supporting institutions.

Subscriptions, orders for back numbers, and changes of address should be sent to Pacific Journal of Mathematics, 103 Highland Boulevard, Berkeley, California, 94708.

PUBLISHED BY PACIFIC JOURNAL OF MATHEMATICS, A NON-PROFIT CORPORATION Printed at Jerusalem Academic Press, POB 2390, Jerusalem, Israel.

\section{Copyright (C) 1976 Pacific Journal of Mathematics} All Rights Reserved 


\section{Pacific Journal of Mathematics}

\section{Vol. 66, No. 2 December, 1976}

Gerald A. Beer, Tax structures whose progressivity is inflation neutral..... 305

William M. Cornette, A generalization of the unit interval............. 313

David E. Evans, Unbounded completely positive linear maps on

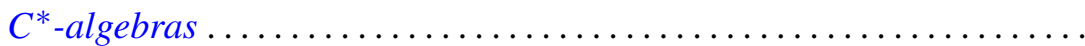

Hector O. Fattorini, Some remarks on convolution equations for

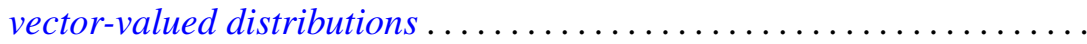

Amassa Courtney Fauntleroy, Automorphism groups of unipotent groups of

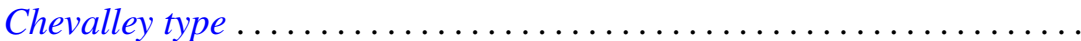

Christian C. Fenske and Heinz-Otto Peitgen, On fixed points of zero index in

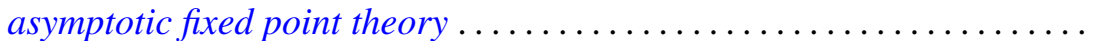

Atsushi Inoue, On a class of unbounded operator algebras. II ............

Herbert Meyer Kamowitz, The spectra of endomorphisms of algebras of

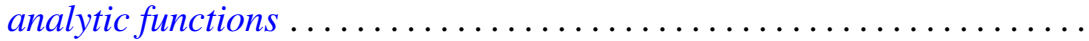

Jimmie Don Lawson, Embeddings of compact convex sets and locally compact cones ....................................

William Lindgren and Peter Joseph Nyikos, Spaces with bases satisfying certain order and intersection properties .....................

Emily Mann Peck, Lattice projections on continuous function spaces ...... 477

Morris Marden and Peter A. McCoy, Level sets of polynomials in $n$ real variables...

Francis Joseph Narcowich, An imbedding theorem for indeterminate Hermitian moment sequences......................

John Dacey O'Neill, Rings whose additive subgroups are subrings ...

Chull Park and David Lee Skoug, Wiener integrals over the sets bounded by sectionally continuous barriers .....................

Vladimir Scheffer, Partial regularity of solutions to the Navier-Stokes equations.

Eugene Spiegel and Allan Trojan, On semi-simple group algebras. II

Katsuo Takano, On Cameron and Storvick's operator valued function space integral 